TRANSACTIONS OF THE

AMERICAN MATHEMATICAL SOCIETY

Volume 353, Number 10, Pages 4039-4056

S 0002-9947(01)02767-2

Article electronically published on June 1, 2001

\title{
A SHARP BOUND ON THE SIZE OF A CONNECTED MATROID
}

\author{
MANOEL LEMOS AND JAMES OXLEY
}

\begin{abstract}
This paper proves that a connected matroid $M$ in which a largest circuit and a largest cocircuit have $c$ and $c^{*}$ elements, respectively, has at most $\frac{1}{2} c c^{*}$ elements. It is also shown that if $e$ is an element of $M$ and $c_{e}$ and $c_{e}^{*}$ are the sizes of a largest circuit containing $e$ and a largest cocircuit containing $e$, then $|E(M)| \leq\left(c_{e}-1\right)\left(c_{e}^{*}-1\right)+1$. Both these bounds are sharp and the first is proved using the second. The second inequality is an interesting companion to Lehman's width-length inequality which asserts that the former inequality can be reversed for regular matroids when $c_{e}$ and $c_{e}^{*}$ are replaced by the sizes of a smallest circuit containing $e$ and a smallest cocircuit containing $e$. Moreover, it follows from the second inequality that if $u$ and $v$ are distinct vertices in a 2-connected loopless graph $G$, then $|E(G)|$ cannot exceed the product of the length of a longest $(u, v)$-path and the size of a largest minimal edge-cut separating $u$ from $v$.
\end{abstract}

\section{INTRODUCTION}

At the 1991 Seattle conference on graph minors, Robin Thomas informally asked the question as to whether every sufficiently large connected matroid has a big circuit or a big cocircuit. This question was rapidly answered at that meeting by Lovász, Schrijver, and Seymour (see [9]) who proved the following result.

1.1. Theorem. Let $M$ be a connected matroid with at least two elements. If a largest circuit of $M$ has c elements and a largest cocircuit has $c^{*}$ elements, then $M$ has at most $2^{c+c^{*}-1}$ elements.

An affirmative answer to Thomas's question can also be obtained from a result of Tuza [15] for set systems. In response to Theorem 1.1, a natural question is whether the bound in the theorem can be improved, particularly if attention is restricted to certain special classes of matroids. For graphic matroids, the following dramatic improvement was found by Pou-Lin Wu [16].

1.2. Theorem. Let $G$ be a loopless 2-connected graph in which a largest cycle has $c$ edges and a largest bond has $c^{*}$ edges. Then $G$ has at most $\frac{1}{2} c c^{*}$ edges.

Of course, $c$ is just the circumference of the graph $G$. There is a wide gap between the graph bound in Theorem 1.2 and the general matroid bound in Theorem 1.1 Reid [11 partially closed this gap. He defined the matroid Ramsey number $n(s, t)$ to be the least positive integer $n$ so that every $n$-element connected matroid has a circuit of size at least $s$ or a cocircuit of size at least $t$. Reid showed that $n(s, t)$

Received by the editors April 12, 1999 and, in revised form, January 18, 2000.

2000 Mathematics Subject Classification. Primary 05B35; Secondary 05C35. 
has several attractive properties that are analogous to properties of the classical Ramsey numbers for graphs established by Erdős and Szekeres [3]. Using these, Reid proved the following improvement of Theorem 1.1

1.3. Theorem. If $M$ is a connected matroid in which a largest circuit has size $c$ and a largest cocircuit has size $c^{*}$, then

$$
|E(M)| \leq\left(\begin{array}{c}
c+c^{*}-2 \\
c-1
\end{array}\right)-2
$$

provided both $c$ and $c^{*}$ exceed three.

Reid [11] and Hurst and Reid [5] also determined some small matroid Ramsey numbers including all $n(s, t)$ with $s$ or $t$ at most three.

The purpose of this paper is to prove that the bound that Pou-Lin Wu proved for graphic matroids also holds for matroids in general. The following is our main result.

1.4. Theorem. Let $M$ be a connected matroid with at least two elements. If a largest circuit of $M$ has c elements and a largest cocircuit has $c^{*}$ elements, then

$$
|E(M)| \leq\left\lfloor\frac{c c^{*}}{2}\right\rfloor .
$$

This theorem solves a problem raised by Oxley [10] and settles a conjecture of Bonin, McNulty, and Reid [1]. For comparison, a lower bound on $|E(M)|$ in terms of $c$ and $c^{*}$ that holds for all matroids $M$ having non-zero rank and corank is

$$
c+c^{*}-2 \leq|E(M)| \text {. }
$$

To see this, observe that $c \leq r(M)+1$ and $c^{*} \leq r^{*}(M)+1$. The bound in (11) is sharp. It is attained by, for example, the whirls, all uniform matroids of non-zero rank and corank, and, more generally, by all matroids with both a free element and a cofree element.

The proof of Theorem 1.4 will use another bound that is of independent interest.

1.5. Theorem. Let $M$ be a connected matroid with at least two elements and let $e$ be an element of $M$. If a largest circuit of $M$ containing e has $c_{e}$ elements and a largest cocircuit containing e has $c_{e}^{*}$ elements, then

$$
|E(M)| \leq\left(c_{e}-1\right)\left(c_{e}^{*}-1\right)+1
$$

The last theorem has an attractive similarity to a bound derived from the widthlength inequality 7. 4. In a matroid $M$, let $e$ be an element that is neither a loop nor a coloop. Let $\lambda(M)+1$ and $\omega(M)+1$ be the cardinalities of a smallest circuit containing $e$ and a smallest cocircuit containing $e$. Lehman [7] showed that if $M$ is regular, then

$$
\lambda(M) \omega(M) \leq|E(M)|-1 .
$$

More generally, it follows from a result of Seymour 12 that the last inequality holds for all binary matroids that have no $F_{7}^{*}$-minor using $e$. In fact, it also holds for $F_{7}^{*}$, although it fails, for example, for $A G(3,2)$.

A straightforward consequence of Theorem[1.5] is the following result for graphs.

1.6. Corollary. Let $u$ and $v$ be distinct vertices in a 2-connected loopless graph $G$. Then $|E(G)|$ cannot exceed the product of the length of a longest $(u, v)$-path and the size of a largest minimal edge-cut separating $u$ from $v$. 
The bounds in Theorems 1.2 1.4 and[1.5 are sharp. Pou-Lin Wu [17 determined all graphs that attain the bound in Theorem 1.2. The cycle matroid of each such graph attains the bound in Theorem 1.4 provided $c$ or $c^{*}$ is even. A non-graphic matroid attaining the bound in Theorem [1.4 which was noted by Reid [11], is $A G(3,2)$. In Section 4, we shall describe all the matroids that attain the bound in Theorem 1.5. noting that there is a close link between these matroids and those found by Wu.

The matroid terminology used here will follow Oxley [9. In particular, the set of circuits of a matroid $M$ will be denoted by $\mathcal{C}(M)$. Theorem 1.5 will be deduced in the next section from a stronger result, while Theorem 1.4 will be proved in Section 3 .

\section{Two strengthenings of Theorem 1.5}

Let $e$ be an element of a matroid $M$ and suppose that $e$ is not a coloop of $M$. We define the circumference of $M$ through $e$ by

$$
c_{e}(M)=\max \{|C|: e \in C \in \mathcal{C}(M)\},
$$

that is, $c_{e}(M)$ is the size of a largest circuit that contains $e$. If $f$ is an element of $M$ that is not a loop, we define $c_{f}^{*}(M)=c_{f}\left(M^{*}\right)$. The circumference $c(M)$ of a non-free matroid is the size of a largest circuit of $M$. If $r(M)>0$, we define $c^{*}(M)=c\left(M^{*}\right)$.

Let $C$ be a circuit of a matroid $M$. A subset $P$ of $E(M)-C$ is a chord of $C$ if $P \in \mathcal{C}(M / C)-\mathcal{C}(M)$. Equivalently, $P$ is a chord of $C$ if and only if $P$ is disjoint from $C$ and $M \mid(C \cup P)$ is a connected matroid having corank two. In particular, when $P$ is a chord of $C$, it is a series class of $M \mid(C \cup P)$.

The first strengthening of Theorem 1.5 will require some more preliminaries. Let $L$ be a subset of the ground set of a matroid $M$ such that $L$ is the union of a set of circuits of $M$ and $r^{*}(M \mid L)=2$. Then $L$ is what Tutte [14 has called a "line" of $M$. We shall call $L$ a Tutte-line since the word "line" is also commonly used in matroid theory to mean a rank- 2 flat. It is not difficult to see that every Tutte-line $L$ of a matroid $M$ has a canonical partition $\left\{L_{1}, L_{2}, \ldots, L_{k}\right\}$ such that a subset $C$ of $L$ is a circuit of $M \mid L$ if and only if $C=L-L_{i}$ for some $i$ in $\{1,2, \ldots, k\}$. A Tutte-line $L$ is connected if $M \mid L$ is a connected matroid. The size of a Tutte-line is the number of sets in its canonical partition. Hence a Tutte-line is connected if and only if it has size at least three.

2.1. Theorem. Let $M$ be a connected matroid such that $|E(M)|>1$ and let $e$ be an element of $M$. Then

$$
|E(M)| \leq c_{e}(M)+\frac{1}{m-2}\left(c_{e}(M)-1\right)\left(c_{e}^{*}(M)-2\right)
$$

for all integers $m$ exceeding two such that every connected Tutte-line of $M$ has size at least $m$.

The dual of this theorem can be restated using the concept of a coline of a matroid, a flat of rank two less than the matroid.

2.2. Corollary. Let $M$ be a connected matroid such that $|E(M)|>1$ and let e be an element of $M$. Suppose that $m$ is an integer exceeding two such that every 
coline of $M$ that is contained in more than two hyperplanes is contained in at least $m$ hyperplanes. Then

$$
|E(M)| \leq c_{e}^{*}(M)+\frac{1}{m-2}\left(c_{e}^{*}(M)-1\right)\left(c_{e}(M)-2\right) .
$$

Proof of Theorem 1.5. In all connected matroids with at least two elements, every connected Tutte-line has size at least three. The theorem follows immediately by taking $m=3$ in Theorem 2.1.

Proof of Corollary 1.6. Add the edge $e$ to $G$ so that it joins $u$ and $v$. Then the result follows immediately from Theorem 1.5 .

Proof of Theorem 2.1. We argue by induction on $|E(M)|$ noting that the result holds when $|E(M)|=2$. Assume it holds for $|E(M)|<k$ and let $|E(M)|=k \geq 3$. Choose a circuit $C$ of $M$ such that $e \in C$ and $|C|=c_{e}(M)$. Observe that the result holds when $E(M)=C$. Thus we may suppose that $E(M) \neq C$. Let $M_{1}, M_{2}, \ldots, M_{n}$ be the connected components of $M / C$.

For each element $f$ of $C-e$, consider the connected component $N_{f}$ of $M /(C-\{e, f\})$ that contains $f$. There is a set $\mathcal{P}_{f}$ of matroids such that

(i) $N_{f}$ is the parallel connection of the matroids in $\mathcal{P}_{f}$ across the basepoint $e$; and

(ii) $N / e$ is connected for every $N$ in $\mathcal{P}_{f}$.

Observe that $\{e, f\}$ is the ground set of a matroid that belongs to $\mathcal{P}_{f}$. For each matroid $N$ in $\mathcal{P}_{f}$, let $C_{N}^{*}$ be a cocircuit of $N$ such that $e \in C_{N}^{*}$ and $\left|C_{N}^{*}\right|=c_{e}^{*}(N)$. Let

$$
C^{*}=\bigcup_{N \in \mathcal{P}_{f}} C_{N}^{*}
$$

Then $C^{*}$ contains $e$ and is a cocircuit of $N_{f}$ and hence of $M$. Thus

$$
c_{e}^{*}(M) \geq\left|C^{*}\right|=1+\sum_{N \in \mathcal{P}_{f}}\left(c_{e}^{*}(N)-1\right) .
$$

For each $f$ in $C-e$, view the set $\mathcal{P}_{f}$ as a multiset and let $\mathcal{P}$ be the multiset that is the union of all these multisets. For each $i$ in $\{1,2, \ldots, n\}$, let $\mathcal{Q}_{i}$ be the multiset of matroids $N$ in $\mathcal{P}$ for which $N / e=M_{i}$. Then $\mathcal{P}$ is the union of the multisets $\mathcal{Q}_{1}, \mathcal{Q}_{2}, \ldots, \mathcal{Q}_{n}$ together with $|C|-1$ copies of $U_{1,2}$, the ground sets of the latter being all sets of the form $\{e, f\}$ such that $f \in C-e$. For all $i$ in $\{1,2, \ldots, n\}$, let $N_{i}$ be a matroid in $\mathcal{Q}_{i}$ for which

$$
c_{e}^{*}\left(N_{i}\right)=\min \left\{c_{e}^{*}(N): N \in \mathcal{Q}_{i}\right\} .
$$

On summing (3) over all $f$ in $C-e$, we get

$$
(|C|-1) c_{e}^{*}(M) \geq(|C|-1)+\sum_{f \in C-e} \sum_{N \in \mathcal{P}_{f}}\left(c_{e}^{*}(N)-1\right) .
$$

Therefore

$$
(|C|-1) c_{e}^{*}(M) \geq(|C|-1)+\left[(|C|-1)+\sum_{i=1}^{n} \sum_{N \in \mathcal{Q}_{i}}\left(c_{e}^{*}(N)-1\right)\right],
$$


where the extra $(|C|-1)$-term appears because, for every $f$ in $C-e$, there is a matroid belonging to $\mathcal{P}_{f}$ having ground set $\{e, f\}$ and this matroid does not belong to any $\mathcal{Q}_{i}$. Hence

$$
(|C|-1)\left(c_{e}^{*}(M)-2\right) \geq \sum_{i=1}^{n}\left|\mathcal{Q}_{i}\right|\left(c_{e}^{*}\left(N_{i}\right)-1\right)
$$

As $|C|=c_{e}(M)$, it follows that

$$
\left(c_{e}(M)-1\right)\left(c_{e}^{*}(M)-2\right) \geq \sum_{i=1}^{n}\left|\mathcal{Q}_{i}\right|\left(c_{e}^{*}\left(N_{i}\right)-1\right) .
$$

Next we obtain, for each $i$ in $\{1,2, \ldots, n\}$, a lower bound on $\left|\mathcal{Q}_{i}\right|$. Let $P_{i}$ be a subset of $E\left(M_{i}\right)$ such that $P_{i} \cup e$ is a circuit of $N_{i}$ of size $c_{e}\left(N_{i}\right)$. Thus

$$
\left|P_{i}\right|=c_{e}\left(N_{i}\right)-1 \text {. }
$$

Moreover, $P_{i}$ is a chord of $C$, so $C \cup P_{i}$ is a connected Tutte-line $L_{i}$ of $M$. Let $\left\{P_{i}, L_{i 1}, L_{i 2}, \ldots, L_{i n_{i}}\right\}$ be the canonical partition of $L_{i}$. Without loss of generality, we may assume that $e \in L_{i n_{i}}$. For all $j$ in $\left\{1,2, \ldots, n_{i}-1\right\}$, the set $L_{i}-L_{i j}$ is a circuit of $M$ containing $e$. Thus

$$
|C| \geq\left|L_{i}-L_{i j}\right|=\left|L_{i}\right|-\left|L_{i j}\right|
$$

But

$$
|C|=\left|L_{i}-P_{i}\right|=\left|L_{i}\right|-\left|P_{i}\right|
$$

Hence

$$
\left|P_{i}\right| \leq \min \left\{\left|L_{i j}\right|: 1 \leq j \leq n_{i}-1\right\}
$$

For all $f$ in $\bigcup_{j=1}^{n_{i}-1} L_{i j}$, since $M_{i}$ and $N_{f}$ are the connected components of $M / C$ and $M /(C-\{e, f\})$, respectively, containing $P_{i}$, we deduce that $E\left(M_{i}\right) \subseteq E\left(N_{f}\right)$ and therefore $\mathcal{P}_{f}$ contains a member $N$ of $\mathcal{Q}_{i}$. Thus

$$
\left|\mathcal{Q}_{i}\right| \geq \sum_{j=1}^{n_{i}-1}\left|L_{i j}\right| \geq\left(n_{i}-1\right)\left|P_{i}\right|
$$

where the second inequality follows by (9). Moreover, since $C \cup P_{i}$ is a connected Tutte-line of $M$ of size $n_{i}+1$, we have $n_{i}+1 \geq m$. Hence, using (10) and (8), we deduce that

$$
\left|\mathcal{Q}_{i}\right| \geq(m-2)\left|P_{i}\right|=(m-2)\left(c_{e}\left(N_{i}\right)-1\right) .
$$

On combining (7) and (11), we get

$$
\left(c_{e}(M)-1\right)\left(c_{e}^{*}(M)-2\right) \geq \sum_{i=1}^{n}(m-2)\left(c_{e}\left(N_{i}\right)-1\right)\left(c_{e}^{*}\left(N_{i}\right)-1\right) .
$$

As $2 \leq\left|E\left(N_{i}\right)\right|<|E(M)|$, the theorem holds for $N_{i}$. In particular, since every connected Tutte-line of $N_{i}$ has size at least three, it follows that

$$
\left|E\left(N_{i}\right)\right| \leq c_{e}\left(N_{i}\right)+\left(c_{e}\left(N_{i}\right)-1\right)\left(c_{e}^{*}\left(N_{i}\right)-2\right)
$$

But $\left|E\left(M_{i}\right)\right|=\left|E\left(N_{i}\right)\right|-1$, so

$$
\left|E\left(M_{i}\right)\right| \leq\left(c_{e}\left(N_{i}\right)-1\right)\left(c_{e}^{*}\left(N_{i}\right)-1\right)
$$


On combining (12) and (13), we get

$$
\begin{aligned}
\left(c_{e}(M)-1\right)\left(c_{e}^{*}(M)-2\right) & \geq \sum_{i=1}^{n}(m-2)\left|E\left(M_{i}\right)\right| \\
& \geq(m-2) \sum_{i=1}^{n}\left|E\left(M_{i}\right)\right|=(m-2)|E(M)-C| .
\end{aligned}
$$

Rewriting the last inequality, using the fact that $c_{e}(M)=|C|$, we obtain

$$
|E(M)| \leq c_{e}(M)+\frac{1}{m-2}\left(c_{e}(M)-1\right)\left(c_{e}^{*}(M)-2\right)
$$

and the theorem follows by induction.

As an immediate consequence of Corollary 2.2, we have the following result.

2.3. Corollary. Let $M$ be a connected matroid such that $|E(M)|>1$. Suppose that $m$ is an integer exceeding two such that every coline of $M$ that is contained in more than two hyperplanes is contained in at least $m$ hyperplanes. Then

$$
|E(M)| \leq c^{*}(M)+\frac{1}{m-2}\left(c^{*}(M)-1\right)(c(M)-2) .
$$

The bound in the last inequality is sharp, it being attained for every $m \geq 3$ by $U_{n-m+2, n}$ provided $n \geq m-1$.

After the original submission of this paper, Guoli Ding (private communication) gave a short proof of Theorem 1.5. A straightforward modification of his argument yields our second strengthening of Theorem 1.5. This result answers a question of Paul Seymour (private communication).

2.4. Theorem. Let $M$ be a connected matroid with at least two elements and let $e$ be an element of $M$. Then there are $c_{e}(M)-1$ cocircuits of $M$ each containing e such that the union of these cocircuits is $E(M)$.

Proof. We argue by induction on $c_{e}(M)$. If $c_{e}(M)=2$, then $M$ is a uniform matroid having rank one, so $E(M)$ is a cocircuit of $M$ and the result follows. Now suppose that the theorem holds for $c_{e}(M)<n$ and let $c_{e}(M)=n \geq 3$.

Let $C^{*}$ be a cocircuit of $M$ that contains $e$. Clearly $C^{*} \neq E(M)$. By a wellknown result of Tutte [14] (see also [9, Theorem 4.3.1]), for every element $z$ of a connected matroid, the deletion or contraction of $z$ leaves a connected matroid. Thus there is a partition $\{X, Y\}$ of $C^{*}-e$ such that $M \backslash X / Y$ is a connected matroid; call it $N$. As every circuit of $M$ that contains $e$ must also meet $X$ or $Y$, it follows that

$$
c_{e}(N)<c_{e}(M)
$$

Since $C^{*} \neq E(M)$, the matroid $N$ has at least two elements. Thus, by the induction assumption, for some $k \leq c_{e}(N)-1$, there are $k$ cocircuits $C_{1}^{*}, C_{2}^{*}, \ldots, C_{k}^{*}$ of $N$ each containing $e$ such that the union of these cocircuits is $E(N)$. For each $C_{i}^{*}$, there is a cocircuit $D_{i}^{*}$ of $M$ such that $C_{i}^{*}=D_{i}^{*}-X$. Hence $C^{*}, D_{1}^{*}, D_{2}^{*}, \ldots, D_{k}^{*}$ are cocircuits of $M$ each containing $e$ and

$$
E(M)=E(N) \cup X \cup Y \subseteq C_{1}^{*} \cup C_{2}^{*} \cup \cdots \cup C_{k}^{*} \cup C^{*} \subseteq D_{1}^{*} \cup D_{2}^{*} \cup \cdots \cup D_{k}^{*} \cup C^{*} .
$$


Thus we have a family of $k+1$ cocircuits of $M$ that covers $E(M)$. Since

$$
k+1 \leq\left(c_{e}(N)-1\right)+1=c_{e}(N) \leq c_{e}(M)-1,
$$

the result follows by induction.

We observe, from the last proof, that the family of $c_{e}(M)-1$ cocircuits that covers $E(M)$ can be required to include any arbitrarily chosen cocircuit of $M$ that contains $e$.

\section{Proof of Theorem 1.4}

In this section, we shall prove Theorem 1.4. In addition to Theorem 1.5, the proof will use two lemmas and we begin the section by proving them. For a circuit $C$ of a matroid $M$ such that $|C| \geq 2$, we define

$$
c^{*}(C, M)=\max \left\{\left|D^{*}\right|: D^{*} \in \mathcal{C}\left(M^{*}\right) \text { and }\left|D^{*} \cap C\right|=2\right\} .
$$

3.1. Lemma. Suppose that $M$ is a connected matroid having a circuit $C$ such that every connected component of $M / C$ is a circuit. If $|E(M)|>1$, then

$$
|E(M)| \leq|C|+c(M)\left\lceil\frac{c^{*}(C, M)-2}{2}\right\rceil .
$$

Proof. Suppose that $(*)$ is false and choose a counterexample $M$ such that $|E(M)|$ is minimum. We note that $E(M) \neq C$, otherwise $(*)$ holds. Suppose that $M / e$ is connected for some $e$ in $C$. Observe that $|E(M / e)|>1$ because $E(M) \neq C$. As $M / C=(M / e) /(C-e)$, it follows that the result holds for the pair $(M / e, C-e)$, by the choice of $(M, C)$. In particular,

$$
|E(M / e)| \leq|C-e|+c(M / e)\left\lceil\frac{c^{*}(C-e, M / e)-2}{2}\right\rceil .
$$

Observe that $c(M / e) \leq c(M)$ and $c^{*}(C-e, M / e) \leq c^{*}(C, M)$. Hence

$$
|E(M)|-1 \leq|C|-1+c(M)\left\lceil\frac{c^{*}(C, M)-2}{2}\right\rceil
$$

and we obtain the contradiction that $(*)$ holds for $M$. Thus $M / e$ is disconnected for every $e$ in $C$.

For all $e$ in $C$, let $P_{e}$ be a chord of $C$ such that $P_{e}$ is the ground set of a connected component of $M / e$. If possible, choose the chords $P_{e}$ such that $P_{a} \neq P_{b}$ for every 2-element subset $\{a, b\}$ of $C$. Suppose that this choice is impossible. Then $P_{a}=P_{b}$ for some distinct $a$ and $b$ in $C$. Therefore both $P_{a} \cup a$ and $P_{a} \cup b$ are circuits of $M$. Thus if $x \in P_{a}$, then $M$ has a circuit contained in $\left(P_{a}-x\right) \cup\{a, b\}$. Suppose that $\{a, b\}$ is a proper subset of $C$. Then, since $P_{a}-x$ is independent in $M / C$, it follows that $\left(P_{a}-x\right) \cup\{a, b\}$ is independent in $M$; a contradiction. We conclude that $C=\{a, b\}$. Moreover, since, by hypothesis, $P_{a}$ and $P_{b}$ cannot be chosen to be different, $M$ is the cycle matroid of the graph consisting of two vertices that are joined by $a, b$, and a path with edge set $P_{a}$. Thus

$$
\begin{gathered}
|E(M)|=|C|+\left|P_{a}\right|=2+\left|P_{a}\right|, \\
c^{*}(C, M)=3, \text { and } c(M)=\left|P_{a}\right|+1 \text {. Therefore } \\
|C|+c(M)\left\lceil\frac{c^{*}(C, M)-2}{2}\right\rceil=2+c(M)=3+\left|P_{a}\right|
\end{gathered}
$$

and we obtain the contradiction that $(*)$ holds for $M$. 
We now know that if $a$ and $b$ are distinct elements of $C$, then $P_{a} \neq P_{b}$. It follows that $M \mid\left[C \cup\left(\bigcup_{e \in C} P_{e}\right)\right]$ is the cycle matroid of the graph that can be obtained from a cycle with edge set $C$ by adding an edge $e^{\prime}$ in parallel with each edge $e$ and then subdividing $e^{\prime}$ to produce a path with edge set $P_{e}$. Hence if $C^{\prime}=\bigcup_{e \in C} P_{e}$, then $C^{\prime}$ is a circuit of $M$, so

$$
\left|C^{\prime}\right| \leq c(M)
$$

Moreover, $M \backslash C^{\prime}$ is connected. Now, suppose that $D^{*}$ is a cocircuit of $M$ such that $\left|D^{*} \cap C\right|=2$, say $D^{*} \cap C=\{a, b\}$. Observe that $D^{*}$ must intersect $P_{a}$ and $P_{b}$ because $P_{a} \cup a$ and $P_{b} \cup b$ are circuits of $M$. Thus

$$
c^{*}\left(C, M \backslash C^{\prime}\right) \leq c^{*}(C, M)-2 .
$$

By the choice of $M$, inequality $(*)$ holds for the pair $\left(M \backslash C^{\prime}, C\right)$, that is,

$$
\left|E\left(M \backslash C^{\prime}\right)\right| \leq|C|+c\left(M \backslash C^{\prime}\right)\left\lceil\frac{c^{*}\left(C, M \backslash C^{\prime}\right)-2}{2}\right\rceil .
$$

As $c\left(M \backslash C^{\prime}\right) \leq c(M)$, it follows using (15) that

$$
|E(M)|-\left|C^{\prime}\right| \leq|C|+c(M)\left\lceil\frac{c^{*}(C, M)-4}{2}\right\rceil .
$$

Hence

$$
\begin{aligned}
|E(M)| & \leq|C|+c(M)\left\lceil\frac{c^{*}(C, M)-2}{2}\right\rceil+\left|C^{\prime}\right|-c(M) \\
& \leq|C|+c(M)\left\lceil\frac{c^{*}(C, M)-2}{2}\right\rceil .
\end{aligned}
$$

The last inequality, which holds by (14), gives the contradiction that the lemma holds for $M$.

3.2. Lemma. Suppose that $M$ is a connected matroid such that $|E(M)|>1$. If $C$ is a circuit of $M$ such that $|C|=c(M)$, then

$$
|E(M)| \leq c(M)\left\lceil\frac{c^{*}(C, M)}{2}\right\rceil .
$$

Proof. Let $M_{1}, M_{2}, \ldots, M_{n}$ be the connected components of $M / C$. Suppose that (**) is false and choose a counterexample $M$ in which the number of $M_{i}$ that are not circuits is a minimum. This number is non-zero, otherwise we get a contradiction to Lemma 3.1. In particular, $E(M) \neq C$ and we may assume that $M_{1}$ is not a circuit.

Let $P$ be a chord of $C$ that is contained in $E\left(M_{1}\right)$. Fix a circuit $C_{1}$ of $M \mid(C \cup P)$ such that $C_{1}-C=P$. Observe that, since $P$ is a series class of $M \mid(C \cup P)$, this matroid has a circuit $D_{1}$ containing $P \cup\left(C-C_{1}\right)$. Then $\left|C_{1}\right|+\left|D_{1}\right| \leq 2|C|$. Moreover, $\left|C_{1}\right|=|P|+\left|C \cap C_{1}\right|$ and $\left|D_{1}\right| \geq|P|+\left|C-C_{1}\right|$. Therefore

$$
|P|+\left|C \cap C_{1}\right|+|P|+\left|C-C_{1}\right| \leq\left|C_{1}\right|+\left|D_{1}\right| \leq 2|C|,
$$

that is, $2|P|+|C| \leq 2|C|$, so

$$
2|P| \leq|C|
$$


By definition, $P$ is a circuit of the component $M_{1}$ of $M / C$. Thus $P$ is a series class of $(M / C) \backslash\left(E\left(M_{1}\right)-P\right)$. Hence

$$
P \text { is a series class of } M \backslash\left(E\left(M_{1}\right)-P\right) \text {. }
$$

For each 2-element subset $\{x, y\}$ of $C$, let $N_{x y}$ be the component of $M /(C-\{x, y\})$ that contains $\{x, y\}$. Choose a 2-element subset $\{a, b\}$ of $C$ such that $E\left(M_{1}\right)$ is contained in $E\left(N_{a b}\right)$. Then $M_{1}$ is a connected component of $N_{a b} / a$. Thus $N_{a b}$ can be decomposed as the parallel connection of two matroids across $a$ such that, for one of these matroids, $N_{1}$, we have $N_{1} / a=M_{1}$. Among the 2-element subsets $\{a, b\}$ of $C$ for which $E\left(M_{1}\right) \subseteq E\left(N_{a b}\right)$, choose one such that

$$
c_{a}^{*}\left(N_{1}\right)-1 \text { is a minimum. }
$$

Let $P_{1}$ be a chord of $C$ contained in $E\left(M_{1}\right)$ such that $\left|P_{1}\right|=c_{a}\left(N_{1}\right)-1$. By Theorem 1.5, we have that

$$
\left|E\left(M_{1}\right)\right|=\left|E\left(N_{1}\right)\right|-1 \leq\left(c_{a}\left(N_{1}\right)-1\right)\left(c_{a}^{*}\left(N_{1}\right)-1\right) .
$$

Now $M_{1}$ is not a circuit. By (17), $P_{1}$ is a series class of $M \backslash\left(E\left(M_{1}\right)-P_{1}\right)$. Thus $M \backslash\left(E\left(M_{1}\right)-P_{1}\right)$ is the 2 -sum, with basepoint $p$, of a circuit with ground set $P_{1} \cup p$ and a matroid $M_{1}^{\prime}$ with ground set $\left(E(M)-E\left(M_{1}\right)\right) \cup p$. We construct a new matroid $M^{\prime}$ from $M \backslash\left(E\left(M_{1}\right)-P_{1}\right)$ by placing $c_{a}^{*}\left(N_{1}\right)-2$ series classes "in parallel" with $P_{1}$, with each such series class having exactly $\left|P_{1}\right|$ elements. More formally, take $c_{a}^{*}\left(N_{1}\right)-2$ disjoint subsets of size $\left|P_{1}\right|$, each disjoint from $E(M)$, and, for each such subset $X$, take a circuit with ground set $X \cup p$. Then $M^{\prime}$ is obtained by deleting $p$ from the parallel connection, with basepoint $p$, of the collection of matroids consisting of all these circuits, the circuit with ground set $P_{1} \cup p$, and the matroid $M_{1}^{\prime}$. For each 2-element subset $\{x, y\}$ of $C$, let $N_{x y}^{\prime}$ be the component of $M^{\prime} /(C-\{x, y\})$ that contains $\{x, y\}$. Then $E\left(N_{a b}^{\prime}\right)$ contains $P_{1}$. Moreover, $N_{a b}^{\prime}$ can be obtained from $N_{a b}$ by replacing $N_{1}$ by the parallel connection, with basepoint $a$, of $c_{a}^{*}\left(N_{1}\right)-1$ circuits, each having exactly $\left|P_{1}\right|+1$ elements. Thus

$$
\left|E\left(M^{\prime}\right)\right|=|E(M)|-\left|E\left(N_{1}\right)\right|+\left|P_{1}\right|\left(c_{a}^{*}\left(N_{1}\right)-1\right)+1 .
$$

Since $\left|P_{1}\right|=c_{a}\left(N_{1}\right)-1$ and $\left|E\left(M_{1}\right)\right|=\left|E\left(N_{1}\right)\right|-1$, we deduce that

$$
\left|E\left(M^{\prime}\right)\right|-|E(M)|=\left(c_{a}\left(N_{1}\right)-1\right)\left(c_{a}^{*}\left(N_{1}\right)-1\right)-\left|E\left(M_{1}\right)\right| \text {. }
$$

Therefore, by (19), $\left|E\left(M^{\prime}\right)\right| \geq|E(M)|$. Since $M^{\prime} / C$ has fewer components that are not circuits than does $M / C$, it will follow that $(* *)$ holds for the pair $\left(M^{\prime}, C\right)$, provided we can show that

$$
|C|=c\left(M^{\prime}\right)
$$

The proof of the lemma will be completed by proving this together with the inequality

$$
c^{*}\left(C, M^{\prime}\right) \leq c^{*}(C, M) .
$$

Suppose that $c\left(M^{\prime}\right)>|C|$. Then $M^{\prime}$ has a circuit $C^{\prime}$ such that $\left|C^{\prime}\right|>|C|$. Certainly $C^{\prime}$ meets $E\left(M^{\prime}\right)-E(M)$, otherwise $C^{\prime}$ is a circuit of $M$; a contradiction. If $C^{\prime} \subseteq E\left(M^{\prime}\right)-E(M)$, then $C^{\prime}$ is the union of two series classes of $M^{\prime}$ each of which is contained in $E\left(M^{\prime}\right)-E(M)$ and has $\left|P_{1}\right|$ elements. Thus

$$
\left|C^{\prime}\right|=2\left|P_{1}\right| \leq|C|
$$

where the last inequality follows by (16) . This contradiction implies that $C^{\prime}$ meets both $E\left(M^{\prime}\right)-E(M)$ and $E(M) \cap E\left(M^{\prime}\right)$. Thus $C^{\prime}$ contains a series class $P^{\prime}$ of $M^{\prime}$ 
that is contained in $E\left(M^{\prime}\right)-E(M)$. Hence $\left(C^{\prime}-P^{\prime}\right) \cup P_{1}$ is also a circuit of $M^{\prime}$ having the same cardinality as $C^{\prime}$. This is a contradiction because this circuit is also a circuit of $M$. Thus (20) holds.

Next we prove (21). Suppose that $c^{*}\left(C, M^{\prime}\right)>c^{*}(C, M)$. Then there is a 2element subset $\{g, h\}$ of $C$ such that

$$
c_{g h}^{*}\left(C, M^{\prime}\right)>c_{g h}^{*}(C, M)
$$

where

$$
c_{g h}^{*}(C, M)=\max \left\{\left|D^{*}\right|: D^{*} \in \mathcal{C}\left(M^{*}\right) \text { and } D^{*} \cap C=\{g, h\}\right\} .
$$

Then certainly $N_{g h} \neq N_{g h}^{\prime}$. Moreover, if $E\left(M_{1}\right)$ avoids $E\left(N_{g h}\right)$, then $M_{1}$ is a component of $M /(C-\{g, h\})$ and so $N_{g h}=N_{g h}^{\prime}$; a contradiction. Thus $E\left(M_{1}\right)$ meets, and so is contained in, $E\left(N_{g h}\right)$. Hence $P_{1}$ is contained in $E\left(N_{g h}\right)$.

Each of $N_{g h}$ and $N_{g h}^{\prime}$ can be obtained as a parallel connection across $g$ of a certain set of matroids. For $N_{g h}$, this set $\mathcal{S}_{g}$ includes a matroid $N_{1}^{\prime \prime}$ for which $N_{1}^{\prime \prime} / g=M_{1}$. The set $\mathcal{S}_{g}^{\prime}$ for $N_{g h}^{\prime}$ must contain $\mathcal{S}_{g}-\left\{N_{1}^{\prime \prime}\right\}$.

We show next that $P_{1} \cup g$ is a circuit of $N_{1}^{\prime \prime}$. Suppose not. Then $P_{1}$ is a circuit of $M /(C-\{g, h\})$ and hence of $M^{\prime} /(C-\{g, h\})$. It follows from the construction of $M^{\prime}$ that $P_{1}$ is a component of $M^{\prime} /(C-\{g, h\})$ and that the elements of $E\left(M^{\prime}\right)$ $E(M)$ are partitioned into $c_{a}^{*}\left(N_{1}\right)-2$ sets each a $\left|P_{1}\right|$-circuit that is a component of $M^{\prime} /(C-\{g, h\})$. Thus $\mathcal{S}_{g}^{\prime}=\mathcal{S}_{g}-\left\{N_{1}^{\prime \prime}\right\}$ and so $c_{g h}^{*}\left(C, M^{\prime}\right) \leq c_{g h}^{*}(C, M)$; a contradiction. Thus $P_{1} \cup g$ is indeed a circuit of $N_{1}^{\prime \prime}$.

The construction of $M^{\prime}$ now implies that $\mathcal{S}_{g}^{\prime}$ is obtained from $\mathcal{S}_{g}-\left\{N_{1}^{\prime \prime}\right\}$ by adjoining $c_{a}^{*}\left(N_{1}\right)-1$ circuits, each of size $\left|P_{1}\right|+1$. Thus

$$
c_{g h}^{*}\left(C, M^{\prime}\right)-c_{g h}^{*}(C, M)=\left(c_{a}^{*}\left(N_{1}\right)-1\right)-\left(c_{g}^{*}\left(N_{1}^{\prime \prime}\right)-1\right) .
$$

But, by the choice of $N_{1}$, the right-hand side of this equation is not positive. Hence $c_{g h}^{*}\left(C, M^{\prime}\right) \leq c_{g h}^{*}(C, M)$; a contradiction. We conclude that (21) holds.

Since (**) holds for $M^{\prime}$ and $c(M)=|C|=c\left(M^{\prime}\right)$,

$$
\left|E\left(M^{\prime}\right)\right| \leq c(M)\left\lceil\frac{c^{*}\left(C, M^{\prime}\right)}{2}\right\rceil \text {. }
$$

But $|E(M)| \leq\left|E\left(M^{\prime}\right)\right|$ and $c^{*}\left(C, M^{\prime}\right) \leq c^{*}(C, M)$. Hence

$$
|E(M)| \leq c(M)\left\lceil\frac{c^{*}(C, M)}{2}\right\rceil ;
$$

a contradiction.

We are now ready to prove the main result of the paper.

Proof of Theorem 1.4. Evidently, it suffices to prove that

$$
|E(M)| \leq \frac{c(M) c^{*}(M)}{2} .
$$

We shall first prove this when $c^{*}(M)$ is even. By Lemma 3.2.

$$
|E(M)| \leq c(M)\left\lceil\frac{c^{*}(C, M)}{2}\right\rceil,
$$

for any circuit $C$ of $M$ such that $|C|=c(M)$. As $c^{*}(C, M) \leq c^{*}(M)$, it follows that

$$
|E(M)| \leq c(M)\left\lceil\frac{c^{*}(C, M)}{2}\right\rceil \leq c(M)\left\lceil\frac{c^{*}(M)}{2}\right\rceil=\frac{c(M) c^{*}(M)}{2}
$$


where the last equality follows since $c^{*}(M)$ is an even integer.

When $c^{*}(M)$ is odd, let $M^{\prime}$ be the matroid obtained from $M$ by inserting an element in parallel with each element of the latter. Then $c^{*}\left(M^{\prime}\right)=2 c^{*}(M)$. Thus $c^{*}\left(M^{\prime}\right)$ is even and hence

$$
\left|E\left(M^{\prime}\right)\right| \leq \frac{c\left(M^{\prime}\right) c^{*}\left(M^{\prime}\right)}{2} .
$$

As $\left|E\left(M^{\prime}\right)\right|=2|E(M)|$ and $c\left(M^{\prime}\right)=c(M)$, we get

$$
2|E(M)| \leq c(M) c^{*}(M)
$$

and the result follows.

Reid [11] noted that the matroid Ramsey numbers have the property that $n(1,1)$ $=1$ and $n(s, t)=2$ if $s$ or $t$ is in $\{1,2\}$ provided $(s, t) \neq(1,1)$. As a consequence of the last theorem, we can determine $n(s, t)$ for all other pairs $(s, t)$. Before this result, for $s \leq t$, the Ramsey number with $s$ largest for which the exact value was known was $n(6,6)$, which Bonin, McNulty, and Reid [1] showed was equal to 13.

3.3. Corollary. For all integers $s$ and $t$ exceeding two,

$$
n(s, t)=\left\lfloor\frac{(s-1)(t-1)}{2}\right\rfloor+1 .
$$

Proof. It follows immediately from Theorem 1.4 that

$$
n(s, t) \leq\left\lfloor\frac{(s-1)(t-1)}{2}\right\rfloor+1 \text {. }
$$

We shall show that equality holds here by giving, for all $s$ and $t$ exceeding two, an example of a connected matroid $M$ with $c(M)=s-1, c^{*}(M)=t-1$, and

$$
|E(M)|=\left\lfloor\frac{(s-1)(t-1)}{2}\right\rfloor .
$$

In each case, the matroid $M$ will be the cycle matroid of a graph $G$. If $t$ is odd, let $G$ be obtained from a cycle with $s-1$ edges by replacing each edge by $\frac{t-1}{2}$ edges in parallel. If $s$ is odd, let $G$ consist of two vertices joined by $t-1$ internally disjoint paths each of length $\frac{s-1}{2}$. Finally, if $s$ and $t$ are both even, let $G$ be constructed by joining two vertices by three internally disjoint paths, of lengths $\frac{s-2}{2}, \frac{s-2}{2}, \frac{s}{2}$, and then replacing each edge of the first and last paths by $\frac{t-2}{2}$ edges in parallel.

\section{The matroids attaining the bound of Theorem 1.5}

In this section, we characterize the matroids for which equality holds in the bound in Theorem 1.5 In addition, we relate this class of matroids to the graphs that attain the bound in Theorem 1.2 We denote by $\mathcal{B}_{3}$ the class of matroids that attain the bound in Theorem 1.5 that is, those matroids $M$ that have an element $e$ for which

$$
|E(M)|=c_{e}(M)+\left(c_{e}(M)-1\right)\left(c_{e}^{*}(M)-2\right) .
$$

It is straightforward to check that $\mathcal{B}_{3}$ is closed under duality and that every circuit with at least two elements including $e$ belongs to $\mathcal{B}_{3}$. In the next lemma, we shall prove that $\mathcal{B}_{3}$ is closed under series connection, provided some conditions are satisfied. A matroid $M$ is uniform with respect to an element $f$ if every circuit containing $f$ has the same cardinality $d$ and every cocircuit containing $f$ has the 
same cardinality $d^{*}$. A matroid $M$ is a uniform series connection of $k$ matroids $M_{1}, M_{2}, \ldots, M_{k}$ for some $k \geq 2$ if the following hold:

4.1. (i) $M_{1}, M_{2}, \ldots, M_{k}$ are connected matroids each having at least two elements;

(ii) $E\left(M_{i}\right) \cap E\left(M_{j}\right)=\{e\}$ for every 2 -subset $\{i, j\}$ of $\{1,2, \ldots, k\}$;

(iii) $c_{e}^{*}\left(M_{1}\right)=c_{e}^{*}\left(M_{2}\right)=\cdots=c_{e}^{*}\left(M_{k}\right)$;

(iv) $M_{1}, M_{2}, \ldots, M_{k}$ are uniform with respect to e; and

(v) $M$ is $S\left(M_{1}, M_{2}, \ldots, M_{k}\right)$, the series connection with basepoint e of $M_{1}, M_{2}$, $\ldots, M_{k}$.

4.2. Lemma. Let $M_{1}, M_{2}, \ldots, M_{k}$ be matroids satisfying 4.1)(i)-(ii). Let $M=$ $S\left(M_{1}, M_{2}, \ldots, M_{k}\right)$. Then $M \in \mathcal{B}_{3}$ if and only if all of $M_{1}, M_{2}, \ldots, M_{k}$ are in $\mathcal{B}_{3}$ and (4.1)(iii) holds. Moreover, $M$ is uniform with respect to $e$ if and only if (4.1) (iii) and 4.1])(iv) hold.

Proof. Clearly

$$
|E(M)|=1+\sum_{i=1}^{k}\left(\left|E\left(M_{i}\right)\right|-1\right) .
$$

Thus, by applying Theorem 1.5 to each $M_{i}$, we get

$$
\begin{aligned}
|E(M)| & \leq 1+\sum_{i=1}^{k}\left[c_{e}\left(M_{i}\right)-1+\left(c_{e}\left(M_{i}\right)-1\right)\left(c_{e}^{*}\left(M_{i}\right)-2\right)\right] \\
& =1+\sum_{i=1}^{k}\left(c_{e}\left(M_{i}\right)-1\right)+\sum_{i=1}^{k}\left(c_{e}\left(M_{i}\right)-1\right)\left(c_{e}^{*}\left(M_{i}\right)-2\right) \\
& \leq c_{e}(M)+\max \left\{c_{e}^{*}\left(M_{i}\right)-2: 1 \leq i \leq k\right\} \sum_{i=1}^{k}\left(c_{e}\left(M_{i}\right)-1\right) \\
& =c_{e}(M)+\max \left\{c_{e}^{*}\left(M_{i}\right)-2: 1 \leq i \leq k\right\}\left(c_{e}(M)-1\right) \\
& =c_{e}(M)+\left(c_{e}^{*}(M)-2\right)\left(c_{e}(M)-1\right) .
\end{aligned}
$$

Thus $M \in \mathcal{B}_{3}$ if and only if equality occurs throughout the above, that is, if and only if $M_{1}, M_{2}, \ldots, M_{k}$ are all in $\mathcal{B}_{3}$, and

$$
c_{e}^{*}\left(M_{1}\right)=c_{e}^{*}\left(M_{2}\right)=\cdots=c_{e}^{*}\left(M_{k}\right) .
$$

Hence the first part of the lemma holds. The second part follows straightforwardly from the definition of series connection.

The proof of the characterization of the matroids attaining equality in Theorem 1.5] will use two results of Seymour [13]. Because the terminology of that paper is different from that used here, we have translated Seymour's results into our terminology. The first result is [13, (3.7)], restated in the special case that $Z$ is a circuit.

4.3. Lemma. Let $e$ be an element of a circuit $C$ of a connected matroid $M$. If $M \backslash e$ is connected, then there is no partition $\left\{X_{1}, X_{2}\right\}$ of $E(M)-e$ such that

(i) no chord of $C$ meets both $X_{1}$ and $X_{2}$; and

(ii) for each $i$ in $\{1,2\}$, if $P$ is a chord of $C$ contained in $X_{i}$, then $X_{i}$ contains a circuit containing $P$. 
The second lemma is a restatement of a special case of [13, (3.8)].

4.4. Lemma. Let $C$ and $D$ be circuits of a matroid $M$ such that $C \cap D=\{e\}$ and $(C \cup D)-e$ is also a circuit of $M$. Then either $(C \cup D)-e$ has a chord $A$ that is different from $\{e\}$ and is not a chord of $C$ or of $D$, or there is a partition $\left\{X_{1}, X_{2}\right\}$ of $E(M \backslash e)$ such that $X_{1} \supseteq C-e$ and $X_{2} \supseteq D-e$, and

$$
r\left(X_{1}\right)+r\left(X_{2}\right)-r(M \backslash e)=r(C-e)+r(D-e)-r((C \cup D)-e)=1 .
$$

4.5. Theorem. $\mathcal{B}_{3}$ is the minimal class $\mathcal{M}$ of matroids with the following properties.

(i) Every circuit that contains $e$ and has at least two elements is in $\mathcal{M}$.

(ii) The dual of every member of $\mathcal{M}$ is in $\mathcal{M}$.

(iii) The uniform series connection of a collection of members of $\mathcal{M}$ is in $\mathcal{M}$.

Proof. We noted above that $\mathcal{B}_{3}$ contains all circuits with at least two elements including $e$ and that $\mathcal{B}_{3}$ is closed under both duality and uniform series connection. The proof will be completed by proving the following, where the superfluous strength of the assertion will facilitate the argument.

4.5.1. If $M \in \mathcal{B}_{3}$, then $M$ is uniform with respect to $e$, and $M$ or its dual is a circuit or is a uniform series connection of a collection of matroids in $\mathcal{B}_{3}$.

We shall argue by induction on $|E(M)|$ noting that the assertion holds when $M$ is a circuit. Thus we may assume that $M$ is not a circuit.

We shall use the same notation as in the proof of Theorem 2.1 taking $m=3$. When a matroid attains equality in the bound in Theorem 1.5 , equality must hold in all the inequalities that appear in the proof of Theorem 2.1 .

First, observe that, to achieve equality in (6), we must have that, for all $i$ in $\{1,2, \ldots, n\}$,

$$
c_{e}^{*}\left(N_{i}\right)=c_{e}^{*}(N)
$$

for every $N$ in $\mathcal{Q}_{i}$. Thus $N_{i}$ can be chosen arbitrarily in $\mathcal{Q}_{i}$.

Equality must also hold in (9), that is,

$$
\left|P_{i}\right|=\min \left\{\left|L_{i j}\right|: 1 \leq j \leq n_{i}-1\right\},
$$

so, for all $j$ in $\left\{1,2, \ldots, n_{i}-1\right\}$,

$$
\left|L_{i j}\right|=\left|P_{i}\right| \text {. }
$$

Since equality must hold in (10) and (11), we have that, for all $i$ in $\{1,2, \ldots, n\}$,

$$
\left(n_{i}-1\right)\left|P_{i}\right|=\left|\mathcal{Q}_{i}\right|=\left|P_{i}\right| \text {. }
$$

Thus, for all such $i$,

$$
n_{i}=2
$$

As any $N$ in $\mathcal{Q}_{i}$ can be taken to be $N_{i}$, and since equality holds in (13), we have that, for all $N$ in $\mathcal{Q}_{i}$,

Hence

$$
c_{e}(N)-1=\frac{\left|E\left(M_{i}\right)\right|}{c_{e}^{*}(N)-1}=\frac{\left|E\left(M_{i}\right)\right|}{c_{e}^{*}\left(N_{i}\right)-1}=c_{e}\left(N_{i}\right)-1 .
$$

$$
c_{e}\left(N_{i}\right)=c_{e}(N)
$$

for every $N$ in $\mathcal{Q}_{i}$. 
Again, since equality holds in (13),

$$
\left|E\left(M_{i}\right)\right|=\left|E\left(N_{i}\right)\right|-1=\left(c_{e}\left(N_{i}\right)-1\right)\left(c_{e}^{*}\left(N_{i}\right)-1\right),
$$

and so $N_{i} \in \mathcal{B}_{3}$. Thus, by the induction assumption, we deduce the following:

4.5.2. For all $i$ in $\{1,2, \ldots, n\}$, the matroid $N_{i}$ is uniform with respect to $e$.

Next we observe that the chords of $C$ have the following property.

4.5.3. For all $i$ in $\{1,2, \ldots, n\}$, every chord of $C$ contained in $E\left(M_{i}\right)$ has cardinality equal to $\left|P_{i}\right|$.

To see this, we note that, for such a chord $P$, the set $P \cup e$ is a circuit of $N$ for some $N$ in $\mathcal{Q}_{i}$. As this $N$ can be taken to be $N_{i}$, the assertion follows from (8) and (24) because $\left|P_{i}\right|=c_{e}\left(N_{i}\right)-1$ and $N_{i}$ is uniform with respect to $e$.

Next we show the following:

4.5.4. Suppose $i \in\{1,2, \ldots, n\}$. Let $P_{i}^{\prime}$ be a chord of $C$ contained in $E\left(M_{i}\right)$ and let $C_{i}$ and $C_{i}^{\prime}$ be the unique circuits of $M \mid\left(C \cup P_{i}\right)$ and $M \mid\left(C \cup P_{i}^{\prime}\right)$, respectively, that avoid e. Then

$$
C_{i} \cap C=L_{i 1}=C_{i}^{\prime} \cap C .
$$

By definition, $\mathcal{Q}_{i}=\bigcup_{f \in C-e}\left\{N \in \mathcal{P}_{f}: N / e=M_{i}\right\}$. Also $\left|\left\{N \in \mathcal{P}_{f}: N / e=M_{i}\right\}\right|$ is 0 or 1 for all $f$ in $C-e$ and, by (23), $n_{i}=2$. Therefore, from the paragraph between (9) and (10), we have that, for all $f$ in $L_{i 1}$, there is a matroid $N$ in $\mathcal{P}_{f}$ such that $N / e=M_{i}$. Thus

$$
\begin{aligned}
\left|\mathcal{Q}_{i}\right| & =\mid\left\{f \in C-e: \mathcal{P}_{f} \text { contains a matroid } N \text { such that } N / e=M_{i}\right\} \mid \\
& \geq\left|L_{i 1}\right| .
\end{aligned}
$$

But equality holds in (10) and therefore holds in the above. Hence, as $L_{i 1}=C_{i} \cap C$, we have

$$
C_{i} \cap C=\left\{f \in C-e: \mathcal{P}_{f} \text { contains a matroid } N \text { such that } N / e=M_{i}\right\} .
$$

By (4.5.3), $\left|P_{i}^{\prime}\right|=\left|P_{i}\right|$ and so $P_{i}^{\prime}$ can be used in place of $P_{i}$ throughout the proof of Theorem 2.1 and in the above. In particular,

$$
C_{i}^{\prime} \cap C=\left\{f \in C-e: \mathcal{P}_{f} \text { contains a matroid } N \text { such that } N / e=M_{i}\right\} .
$$

We conclude that

$$
C_{i} \cap C=C_{i}^{\prime} \cap C,
$$

that is, (4.5.4) holds.

On combining (4.5.4) and (22), we get that

$$
\left|C_{i} \cap C\right|=\left|P_{i}\right| .
$$

We now show that two sets $C_{i} \cap C$ and $C_{j} \cap C$ are either disjoint or are comparable.

4.5.5. If $i$ and $j$ are distinct elements of $\{1,2, \ldots, n\}$, then

(i) $C_{i} \cap C_{j}=\emptyset$; or

(ii) $C_{i} \cap C \subseteq C_{j} \cap C$; or

(iii) $C_{j} \cap C \subseteq C_{i} \cap C$. 
By (23),$n_{i}=n_{j}=2$. Thus each of the Tutte-lines $C \cup P_{i}$ and $C \cup P_{j}$ has size three, so the matroids $M \mid\left(C \cup P_{i}\right)$ and $M \mid\left(C \cup P_{j}\right)$ are binary. It is a straightforward consequence of a result of Lemos [8] (3.1)] that $M \mid\left(C \cup P_{i} \cup P_{j}\right)$ is also binary. Assume that none of (i)-(iii) holds. Then $M \mid\left(C \cup P_{i} \cup P_{j}\right)$ is the cycle matroid of the graph obtained from a cycle $C$ by adding two disjoint paths $P_{i}$ and $P_{j}$ each with both endpoints on $C$ such that the resulting graph is a subdivision of $K_{4}$. Let $C^{\prime}=P_{i} \cup P_{j} \cup\left(C_{i} \cap C_{j} \cap C\right) \cup\left(C-\left(C_{i} \cup C_{j}\right)\right)$. Then $C^{\prime}$ is a circuit of $M$ containing $e$ and

$$
\left|C^{\prime}\right|=\left|P_{i}\right|+\left|P_{j}\right|+\left|C_{i} \cap C_{j} \cap C\right|+\left|C-\left(C_{i} \cup C_{j}\right)\right| .
$$

Thus, by (26) and the fact that $\left\{C_{i} \cap C_{j} \cap C, C-\left(C_{i} \cup C_{j}\right),\left(C_{i} \cap C\right)-C_{j},\left(C_{j} \cap C\right)-C_{i}\right\}$ is a partition of $C$, we deduce that

$$
\left|C^{\prime}\right|=\left|C_{i} \cap C\right|+\left|C_{j} \cap C\right|+|C|-\left|\left(C_{i} \cap C\right)-C_{j}\right|-\left|\left(C_{j} \cap C\right)-C_{i}\right| .
$$

As $C_{i} \cap C$ properly contains $\left(C_{i} \cap C\right)-C_{j}$, and $C_{j} \cap C$ properly contains $\left(C_{j} \cap C\right)-C_{i}$, we deduce that $\left|C^{\prime}\right|>|C|$; a contradiction. We conclude that (4.5.5) holds.

Let $Q_{1}, Q_{2}, \ldots, Q_{k}$ be the distinct maximal sets in the family $\left\{C_{i} \cap C: 1 \leq i \leq\right.$ $n\}$. Then, by (4.5.5), $Q_{i} \cap Q_{j}=\emptyset$ for all 2-element subsets $\{i, j\}$ of $\{1,2, \ldots, k\}$. For each $j$ in $\{1,2, \ldots, k\}$, let $Q_{j}^{\prime}$ be the union of all $E\left(M_{i}\right)$ for which $C_{i} \cap C \subseteq Q_{j}$. Then $\left\{Q_{1}^{\prime}, Q_{2}^{\prime}, \ldots, Q_{k}^{\prime}\right\}$ is a partition of $E(M)-C$. Letting $X_{1}=Q_{1} \cup Q_{1}^{\prime}$ and $X_{2}=E(M \backslash e)-\left(Q_{1} \cup Q_{1}^{\prime}\right)$, we deduce from Lemma 4.3 that either (a) $M \backslash e$ is disconnected, or (b) $X_{2}$ is empty. In the first case, $M$ is a series connection with basepoint $e$ of a set of connected matroids each having at least two elements. Since $M \in \mathcal{B}_{3}$, it follows by Lemma 4.2 and the induction assumption that $M$ satisfies (4.5.1). Thus we may assume that (ii) holds. Hence

$$
k=1 \text { and } Q_{1}=C-e .
$$

Moreover, for some $i$ in $\{1,2, \ldots, n\}$, we have $Q_{1}=C_{i} \cap C$, and, by (26), $\left|C_{i} \cap C\right|=$ $\left|P_{i}\right|$. Hence $\left|P_{i}\right|=|C|-1$ and so, letting $D=P_{i} \cup e$, we have that $D$ is a circuit of $M$ containing $e$ and having cardinality $|C|$, and $C \cup D$ is a Tutte-line of size three for which the canonical partition is $\{\{e\}, C-e, D-e\}$. Thus $(C \cup D)-e$ is a circuit of $M$.

Next we shall prove the following:

4.5.6. Let $A$ be a chord of $(C \cup D)-e$. Then $A=\{e\}$, or $A$ is a chord of $C$ or $D$.

Suppose that $A \neq\{e\}$ and let $W=M \mid(C \cup D \cup A)$. The proof of (4.5.6) will be broken into several steps the first of which is as follows.

4.5.7. If $D_{1}$ and $D_{2}$ are circuits of $W$ and $e \in D_{1} \cap D_{2}$, then $D_{1} \cup D_{2}$ is a proper subset of $E(W)$.

Suppose that $E(W)=D_{1} \cup D_{2}$. Then

$$
\begin{aligned}
\left|D_{1} \cup D_{2}\right| & =|E(W)|=|C \cup D \cup A|=|C|+|D-e|+|A| \\
& =2|C|-|\{e\}|+|A| \geq 2|C| .
\end{aligned}
$$

As $e \in D_{1} \cap D_{2}$, it follows that $\left|D_{1} \cap D_{2}\right| \geq 1$. Thus

$$
\left|D_{1}\right|+\left|D_{2}\right|=\left|D_{1} \cup D_{2}\right|+\left|D_{1} \cap D_{2}\right| \geq 2|C|+1 \text {. }
$$

Therefore $\left|D_{1}\right|>|C|$ or $\left|D_{2}\right|>|C|$. This is a contradiction because $|C|=c_{e}(M)$ and $e \in D_{1} \cap D_{2}$. We conclude that (4.5.7) holds. 
Now consider $W^{*}$, which has rank three since $W$ has corank three. Recall that the points and lines of a matroid are its rank-1 and rank-2 flats.

4.5.8. $W^{*}$ has the following properties:

(i) $\{e\}$ and $A$ are points of $W^{*}$.

(ii) If $L_{1}$ and $L_{2}$ are lines of $W^{*}$ such that $e \notin L_{1} \cup L_{2}$, then $L_{1} \cap L_{2} \neq \emptyset$.

(iii) There are exactly three lines in $W^{*}$ that contain $A$.

(iv) The line that contains $e$ and $A$ does not contain another point.

Part (i) holds because $\{e\}$ and $A$ are series classes of $W$. Part (ii) follows immediately from (4.5.7) by duality. Parts (iii) and (iv) hold because $E(W)-A$ contains exactly three circuits, and because $E(W)-(A \cup e)$ is the circuit $(C \cup D)-e$ of $W$. Thus (4.5.8) holds.

By (4.5.8) (iii), $W^{*}$ has exactly three lines, say $L_{1}, L_{2}$, and $L_{3}$, that contain $A$. By (4.5.8) (iv), we may suppose that $L_{1}=A \cup e$. Next we establish the following:

4.5.9. $L_{2}$ or $L_{3}$ contains just one point different from $A$.

For each $i$ in $\{2,3\}$, let $p_{i 1}$ and $p_{i 2}$ be distinct points contained in $L_{i}-A$. For each $j$ and $k$ in $\{1,2\}$, let $K_{j k}$ be the line of $W^{*}$ that contains $p_{2 j}$ and $p_{3 k}$. Suppose that, for some $i, j, k$, and $l$ in $\{1,2\}$ such that $i \neq k$ and $j \neq l$, we have $e \notin K_{i j} \cup K_{k l}$. Then, by 4.5.8) (ii), $K_{i j} \cap K_{k l}=\{p\}$ for some point $p$. Clearly $p$ is not on $L_{2}$ or $L_{3}$ and, by 4.5.8) (iv), it is not on $L_{1}$. Hence $p$ does not exist. Thus $K_{i j}$ or $K_{k l}$ has $e$ as a point. Hence $e$ is on $K_{11}$ or $K_{22}$, and it is on $K_{21}$ or $K_{12}$. Thus we have two distinct lines having two common points. This contradiction implies that (4.5.9) holds.

By 4.5.9, we may suppose that $L_{2}-A$ contains a single point $Z$. Then $Z \cup e$ is the complement of $L_{3}$ and so is a circuit of $W$ that does not intersect $A$. Hence $Z \cup e$ is $C$ or $D$. Moreover, $A$ is a component of $W^{*} \backslash[(C \cup D)-Z]$ and hence of $W /[(C \cup D)-Z]$. Thus $A$ is a component of $[M \mid(C \cup D \cup A)] /[(C \cup D)-Z]$. But $A$ is a circuit of $[M \mid(C \cup D \cup A)] /[C \cup D]$. Hence $A$ is a circuit of $M /[(C \cup D)-Z]$, that is, $A$ is a chord of $(C \cup D)-Z$. Hence $A$ is a chord of $C$ or $D$, and (4.5.6) holds.

By (4.5.6) and Lemma 4.4, it follows that there is a partition $\left\{X_{1}, X_{2}\right\}$ of $E(M \backslash e)$ such that $X_{1} \supseteq C-e$ and $X_{2} \supseteq D-e$, and $r\left(X_{1}\right)+r\left(X_{2}\right)-r(M \backslash e)=1$. Since $e$ is in the closure of both $X_{1}$ and $X_{2}$, it follows that $M$ is the parallel connection, with basepoint $e$, of matroids with ground sets $X_{1} \cup e$ and $X_{2} \cup e$. Thus $M^{*}$ is a series connection, with basepoint $e$, of two matroids. Since $\mathcal{B}_{3}$ is closed under duality, the theorem follows using Lemma 4.2 and the induction assumption.

Evidently each of the matroids that attains the bound in Theorem 1.5 is a series-parallel network. The following result is thus an immediate consequence of Theorems 1.5 and 4.5

4.6. Corollary. Let $M$ be a connected matroid that is not a series-parallel network. If $e \in E(M)$, then

$$
|E(M)| \leq\left(c_{e}(M)-1\right)\left(c_{e}^{*}(M)-1\right) .
$$

We conclude by noting an interesting link between the matroids attaining the bound in Theorem 1.5 and the graphs attaining the bound in Theorem 1.2. The following result was proved by $\mathrm{Wu}[17]$. 
4.7. Theorem. Let $M$ be a connected graphic matroid with at least two elements. Then $E(M)=\frac{1}{2} c(M) c^{*}(M)$ if and only if, for some $k \geq 2$, there is a sequence of positive integers $\left(m, n_{1}, n_{2}, \ldots, n_{k}\right)$ for which $n_{i} \leq \frac{1}{2} \sum_{j=1}^{k} n_{j}$ for all $i$ in $\{1,2$, $\ldots, k\}$ such that either $M$ or $M^{*}$ can be obtained by deleting e from the parallel connection, with basepoint $e$, of $k$ matroids $M_{1}, M_{2}, \ldots, M_{k}$, where each $M_{i}$ is the cycle matroid of a graph that can be obtained from a 2-cycle containing e by a sequence of the following operations:

(a) in a cycle containing e, replacing every edge except e by two edges in parallel; and

(b) in a bond containing e, subdividing every edge except e exactly once;

where this sequence consists of $n_{i}-1$ operations of type (a) and $m-1$ operations of type (b).

Using Theorem 4.5] one can show that the matroids in $\mathcal{B}_{3}$ can be characterized in a similar way. To prevent the paper from becoming too long, we omit the details of the proof.

4.8. Corollary. Let $M$ be a connected matroid with at least two elements and suppose $e \in E(M)$. Then $|E(M)|=\left(c_{e}(M)-1\right)\left(c_{e}^{*}(M)-1\right)+1$ if and only if, for some $k \geq 1$, there is a sequence of positive integers $\left(m, n_{1}, n_{2}, \ldots, n_{k}\right)$ such that $M$ is the parallel connection, with basepoint $e$, of $k$ matroids $M_{1}, M_{2}, \ldots, M_{k}$, where each $M_{i}$ is the cycle matroid of a graph that can be obtained from a 2-cycle containing e by a sequence of the following operations:

(a) in a cycle containing e, replacing every edge except e by two edges in parallel; and

(b) in a bond containing e, subdividing every edge except e exactly once;

where this sequence consists of $n_{i}-1$ operations of type (a) and $m-1$ operations of type (b).

\section{ACKNOWLEDGEMENTS}

The authors thank Guoli Ding for showing them a short proof of Theorem 1.5 The first author was partially supported by CNPq, CAPES, FINEP, and PRONEX $107 / 97$. The second author was partially supported by the National Security Agency.

\section{REFERENCES}

[1] Bonin, J., McNulty, J., and Reid, T.J., The matroid Ramsey number $n(6,6)$, Combin. Probab. Comput. 8(1999), 229-235. MR 2000i:05040

[2] Denley, T. and Reid, T.J., On the number of elements in matroids with small circuits and small cocircuits, Combin. Probab. Comput. 8 (1999), 529-537. CMP 2000:09

[3] Erdős, P. and Szekeres, G., A combinatorial problem in geometry, Compositio Math. 2 (1935), 463-470.

[4] Fulkerson, D.R., Networks, frames, blocking sysyems, in Mathematics of the Decision Sciences, Part 1, Lectures in Applied Math. Vol. 11, Amer. Math. Soc., Providence, R.I., 1968, pp. 303-334. MR 42:4329

[5] Hurst, F. and Reid, T.J., Some small circuit-cocircuit Ramsey numbers for matroids, Combin. Probab. Comput. 4 (1995), 67-80. MR 96d:05033

[6] Hurst, F. and Reid, T.J., Ramsey numbers for cocircuits in matroids, Ars Combin. 45 (1997), 181-192. MR 97a:05067

[7] Lehman, A., On the width-length inequality, Math. Programming 17 (1979), 403-417. MR 83a:94034a 
[8] Lemos, M., k-elimination property for circuits of matroids, J. Combin. Theory Ser. B 51 (1991), 211-226. MR 92e:05027

[9] Oxley, J.G., Matroid Theory, Oxford University Press, New York, 1992. MR 94d:05033

[10] Oxley, J.G., Unavoidable minors in graphs and matroids, in Graph theory and combinatorial biology (Balatonlelle, 1996), Bolyai Soc. Math. Stud. 7, János Bolyai Math. Soc., Budapest, 1999, pp. 279-305. MR 2000c:05038

[11] Reid, T.J., Ramsey numbers for matroids, Europ. J. Combin 18 (1997), 589-595. MR 98h:05139

[12] Seymour, P.D., The matroids with the max-flow min-cut property, J. Combin. Theory Ser. B 23 (1977), 189-222. MR 57:2960

[13] Seymour, P.D., Decomposition of regular matroids, J. Combin. Theory Ser. B 28 (1980), 305-354. MR 82j:05046

[14] Tutte, W.T., Lectures on matroids, J. Res. Nat. Bur. Standards Sect. B 69B (1965), 1-47. MR 31:4023

[15] Tuza, Z., On two intersecting set systems and $k$-continuous Boolean functions, Discrete Appl. Math 16 (1987), 183-185. MR 88a:05007

[16] Wu, P.-L., An upper bound on the number of edges of a 2-connected graph, Combin. Probab. Comput. 6 (1997), 107-113. MR 97m:05146

[17] Wu, P.-L., Extremal graphs with prescribed circumference and cocircumference, Discrete Math. 223 (2000), 299-308. CMP 2001:01

Departamento de Matemática, Universidade Federal de Pernambuco, Recife, PerNAMBUCO 50740-540, BRAZIL

E-mail address: manoel@dmat.ufpe.br

Department of Mathematics, Louisiana State University, Baton Rouge, Louisiana 70803-4918

E-mail address: oxley@math.lsu.edu 